\title{
Phenylacetic Acid (3S,6S)-6-Acetoxy-8-methyl-8-aza-bicyclo[3.2.1]oct-3-yl Ester
}

\section{Yin-Yao Niu and Yang Lu*}

Department of Chemistry, Shanghai Second Medical University, Shanghai-200025, People's Republic of China

Tel. +86 21 63846590-776464, Fax. +86 21 53065329, E-mail: huaxue@shsmu.edu.cn and luyangssmu@yahoo.com

\section{Received: 22 February 2003 / Accepted 22 May 2003 / Published 1 June 2003}

The fact that the configuration of derivatives of acetic acid 8-methyl-8-aza-bicyclo[3.2.1]oct-6-yl ester was considered having significant influence on the muscarinic activity [1] prompted us to synthesize the chiral compounds with the skeleton in order to study the structure-activity relationship.

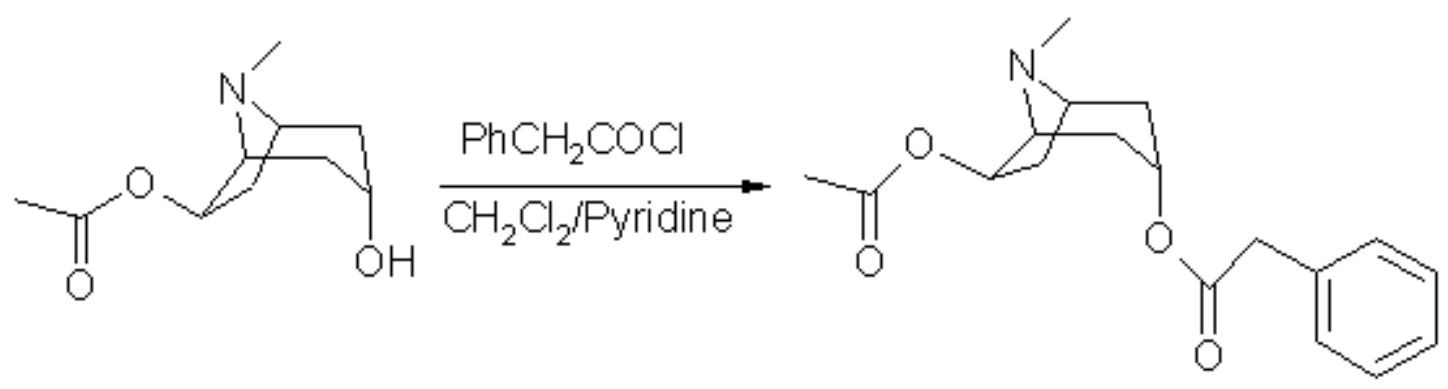

To a solution of (-)-acetic acid (3S,6S)-3-hydroxy-8-methyl-8-aza-bicyclo[3.2.1] oct-6-yl ester [2, 3] $(0.22$ $\mathrm{g}, 1.1 \mathrm{mmol})$ in dichloromethane $(2 \mathrm{~mL})$ with pyridine $(0.1 \mathrm{~mL})$ was added dropwise phenylacetyl chloride $(0.23 \mathrm{~mL}, 1.7 \mathrm{mmol})$. The mixture was stirred for $24 \mathrm{~h}$ at room temperature. The solution was evaporated in vacuo to dryness. The residue was dissolved in water $(5 \mathrm{~mL})$ and basified with concentrated aqueous ammonia. The aqueous solution was extracted with chloroform $(5 \times 6 \mathrm{~mL})$, and the combined organic layers were dried over anhydrous sodium sulfate, filtered, evaporated in vacuo, to give phenylacetic acid (3S,6S)-6-acetoxy-8-methyl-8-aza-bicyclo[3.2.1]oct-3-yl ester $(0.34 \mathrm{~g}, 95 \%)$ as a pale yellow oil.

$[\mathrm{a}]_{\mathrm{D}}^{20}=+2.0^{\circ}\left(\mathrm{c} 1.05, \mathrm{CHCl}_{3}\right)$.

IR (KBr): 2947, 2858, 1732, 1604.

${ }^{1} \mathrm{H}$ NMR (400 MHz, CDCl 3$): 1.64-2.15(\mathrm{~m}, 6 \mathrm{H}), 2.03(\mathrm{~s}, 3 \mathrm{H}), 2.44(\mathrm{~s}, 3 \mathrm{H}), 3.08(\mathrm{~s}, 1 \mathrm{H}), 3.21(\mathrm{~m}, 1 \mathrm{H})$, $3.61(\mathrm{~s}, 2 \mathrm{H}), 4.95(\mathrm{~m}, 1 \mathrm{H}), 5.21(\mathrm{dd}, \mathrm{J}=3.1,7.6 \mathrm{~Hz}, 1 \mathrm{H}), 7.20-7.36(\mathrm{~m}, 5 \mathrm{H})$.

MS: m/z(\%) $317\left(\mathrm{M}^{+}, 19.58\right), 182(38.54), 122(53.49), 94(100), 82(9.95), 43(15.63)$.

Anal. Calc for $\mathrm{C}_{18} \mathrm{H}_{23} \mathrm{NO}_{4}$ : C 68.14, H 7.26, N 4.42. Found C 67.76, H 7.26, N 4.32.

\section{Acknowledgement}

The financial support of Shanghai Municipal Educational Committee is acknowledged.

\section{References and Notes}

1. Lu, Y.; Chen, Z. N. Acta Universitatis Medicinalis Secondae Shanghai 1996, 16, 1.

2. Yang, L. M.; Wang, H. N.; Yan, Z. H. J. Shanghai Second Medical University 1999, 11, 51. 
3. Yan, Z. H.; Lu, Y.; Valler, A.; Liu, H. Z.; Chen, Z. N. Acta Universitatis Medicinalis Secondae Shanghai 2001, 21, 199.

Sample Availability: Available from the authors and from MDPI.

(C) 2003 MDPI. All rights reserved. 